Abanico Veterinario. Enero-Diciembre 2020; 10(1):1-16. http://dx.doi.org/10.21929/abavet2020.7

Artículo Original. Recibido: 21/01/2020. Aceptado: 25/04/2020. Publicado: 03/05/2020.

\title{
Residuos de plaguicidas en miel y cera de colonias de abejas de La Comarca Lagunera
}

\author{
Pesticides residues in honey and wax from bee colonies in La Comarca Lagunera
}

\section{Vargas-Valero Azucena ${ }^{1,2^{*} \underline{\mathrm{D}}}$ Reyes-Carrillo José2i⿺ Véliz-Deras Francisco ${ }^{2 i \underline{D}}$ Gaspar-Ramírez Octavio ${ }^{3 i}$, Rodríguez-Martínez Rafael ${ }^{2 * i[}$}

${ }^{1}$ Instituto Nacional de Investigaciones, Forestales, Agrícolas y Pecuarias. México. ${ }^{2}$ Universidad Autónoma Agraria Antonio Narro-Unidad Laguna. México. ${ }^{3}$ Centro de Investigación y Asistencia en Tecnología y Diseño del Estado de Jalisco, A.C. México. *Autor responsable y de correspondencia: Rodríguez-Martínez Rafael. Carretera a Sta. Fe y Periférico Raúl López Sánchez s/n C.P. 27054, Torreón, Coahuila, México. azvalero@yahoo.com.mx, jlreyes54@gmail.com, alejamorsa@hotmail.com,_velizderas@gmail.com, ogramirez@ciatej.mx, rafael.rdz.mtz@gmail.com

\section{RESUMEN}

Las abejas melíferas son importantes para la seguridad alimentaria y el mantenimiento de la biodiversidad. Se ha presentado un colapso de las colonias ocasionado por la exposición a plaguicidas. El objetivo fue determinar y cuantificar la presencia de plaguicidas en miel y cera de colonias de abejas bajo colapso (BC), con (CA) y sin antecedentes de colapso (SA). Se analizaron cinco muestras de miel y cinco de cera de colonias CA y SA, así como dos de miel y de cera BC; las muestras fueron analizadas por LC-QTOF y GCMS/MS. Se detectaron en total 24 plaguicidas en miel y cera. El acetamiprid se encontró en el $100 \%$ de las muestras. En las colonias BC, presentaron en promedio altos niveles de acetamiprid en cera y miel $(0.402$ y $0.633 \mathrm{mg} \mathrm{kg}^{-1}$ respectivamente). Para las colonias CA, los promedios de acetamiprid fueron 0.686 y 0.266 $\mathrm{mg} \mathrm{kg}^{-1}$ para cera y miel respectivamente, en las colonias $\mathrm{SA}$, los promedios del acetamiprid en cera y miel fueron 0.234 y $0.404 \mathrm{mg} \mathrm{kg}^{-1}$ respectivamente. En conclusión, las colonias CA presentaron la mayor diversidad de plaguicidas seguidas por SA y BC. Estos resultados podrían sugerir la participación de los plaguicidas como causa del colapso de las colonias.

Palabras clave: Apis mellifera, Desorden del Colapso de las Colonias, México y QuEChERS.

\begin{abstract}
Honeybees are important for food security and biodiversity preservation. There has been a collapse of the colonies caused by exposure to pesticides. The aim was to determine and quantify the presence of pesticides in honey and wax from bee colonies, under collapse (BC) and with (CA) and without antecedent of collapse (SA). Five honey samples and five colony wax samples were analyzed from colonies CA y SA, as well as two of honey and two wax from colonies CB; samples were analyzed by LC-QTOF and GCMS/MS. 24 pesticides were detected in honey and wax analyzed. Acetamiprid was found in all samples. In colonies, CB the wax and honey had high averages levels of acetamiprid $(0.402$ and $0.633 \mathrm{mg} \mathrm{kg}-1$ respectively). For wax and honey from colonies $C A$, the averages of acetamiprid were 0.686 and $0.266 \mathrm{mg}$ $\mathrm{kg}-1$ respectively. In wax and honey from colonies SA the averages of acetamiprid were 0.234 and 0.404 mg kg-1 respectively. In conclusion, the colonies CA had the greatest diversity of pesticides, followed by the group SA and finally BC. Our results suggest the participation of pesticides as a cause of colony collapse.
\end{abstract}

Keywords: Apis mellifera, Colony Collapse Disorder, México and QuEChERS. 


\section{INTRODUCCIÓN}

Las abejas melíferas son importantes polinizadores de una gran diversidad de cultivos y plantas nativas, la polinización llevada a cabo por estos insectos es necesaria para el 35 $\%$ de los cultivos destinados a la alimentación humana (Ollerton, 2017); sin embargo, a nivel mundial han sufrido una disminución en sus poblaciones, fenómeno llamado el Desorden del Colapso de las Colonias (DCC) (Brutscher et al., 2016). Las características de las colonias colapsadas, son: la muerte parcial o total de la colonia con presencia de abejas muertas dentro o cerca de la colmena, la desaparición parcial o total de la colonia, con el abandono de las reservas de alimento y las crías y el debilitamiento de la colonia mediante un lento desarrollo durante la primavera, bajo condiciones óptimas (SimonDelso et al., 2014).

Aunque se han reportado diversos factores como las probables causas del colapso de las colonias, uno de los más importantes es la exposición a plaguicidas (CalatayudVernich et al., 2018; Sánchez-Bayo et al., 2016; Traynor et al., 2016). Las abejas se exponen a los plaguicidas cuando buscan los recursos néctar-poliníferos, sobre todo si las colonias están ubicadas cerca de áreas agrícolas (O’Neal et al., 2018). Se ha demostrado que la exposición a dosis subletales de plaguicidas puede afectar el comportamiento de la abeja (Balbuena et al., 2015), el pecoreo (Cresswell y Thompson, 2012), su longevidad (Wu et al., 2011), la termorregulación (Tosi et al., 2016); así como su aprendizaje olfativo y memoria (Lu et al., 2014).

Los residuos de plaguicidas pueden acumularse en el pan de abeja, miel y cera (Johnson et al., 2010; Lozano et al., 2019); presentándose en esta última la capacidad de almacenamiento residual de plaguicidas (Benuszak et al., 2017). Por tanto, de un panal contaminado, los residuos pueden transferirse a la miel almacenada, presentando un riesgo para los consumidores; también, el consumo de "miel en panal", como aditivo de alimentos en el tratamiento de frutas, suplemento alimenticio o como saborizante, representan un riesgo para la salud (Wilmart et al., 2016).

En el semidesierto del norte de México, se ha encontrado la presencia de plaguicidas en bajas concentraciones en muestras de miel y cera (Alcántar-Rosales et al., 2016), y se ha reportado para el periodo de 2010 al 2017 una disminución de hasta el 35\% de las colonias de abejas (SIAP, 2018).

Por lo tanto, el objetivo de esta investigación fue determinar y cuantificar la presencia de plaguicidas en muestras de miel y cera de colonias de abejas, con y sin antecedentes de colapso.

\section{MATERIAL Y MÉTODOS}

Muestreo de las matrices de las colmenas. El muestreo se realizó en el semidesierto del norte de México ( $25^{\circ} 05^{\prime}$ y $26^{\circ} 54^{\prime} \mathrm{LN}$ y $101^{\circ} 40^{\prime}$ y $\left.104^{\circ} 45^{\prime} \mathrm{LO}\right)$, entre los meses de 
junio a septiembre de 2017, con base en el padrón de apicultores registrados en el Comité Sistema Producto Apícola de la Región Lagunera A.C., bajo el criterio de contar con al menos de 10 colonias; siendo seleccionados 43 apicultores, de los cuales, mediante un muestreo aleatorio del $20 \%$ de las colonias de cada apiario y bajo condiciones naturales. Se obtuvieron un total de 132 muestras de panal con miel y cera de aproximadamente 12 $\mathrm{cm}^{2}$; de éstas, para su análisis de plaguicidas, se seleccionaron al azar 12 muestras, de las cuales cinco se clasificaron como provenientes de colonias con antecedentes de colapso (CA); cinco como asintomáticas o provenientes de colonias sin antecedentes de colapso (SA), y dos muestras de apiarios que al momento del muestreo sufrieron el colapso (BC). La clasificación se realizó de acuerdo a los datos obtenidos del apicultor y con base en los criterios definidos por Simon-Delso et al. (2014).

Con un cúter desechable se cortó un pedazo de panal con miel y cera de aproximadamente $12 \mathrm{~cm}^{2}$, se colocó en una bolsa de plástico con su respectiva identificación y posteriormente fueron transportadas al laboratorio de Biología de la Universidad Autónoma Agraria Antonio Narro, Unidad Laguna en Torreón, Coahuila, para almacenarlas a $-20^{\circ} \mathrm{C}$, hasta su análisis en el laboratorio del Centro de Investigación y Asistencia en Tecnología y Diseño del Estado de Jalisco, A. C. (CIATEJ), Apodaca, Nuevo León. A cada una de las muestras (miel y cera) se les determinó165 plaguicidas.

Productos químicos y soluciones. Los estándares analíticos de los plaguicidas se obtuvieron de ChemService, Inc. (West Chester, PA, USA): Sigma-Aldrich-Fluka (St. Louis, MO, USA), Sigma-Aldrich-Supelco (Bellefonte, PA, USA), Accustandard (New Haven, CT, USA), y ULTRA Scientific (N. Kingstown, RI, USA). El ácido fórmico (grado MS) y formiato de amonio (base metal traza) se adquirieron en Sigma-Aldrich. El Acetonitrilo grado HPLC y el agua grado HPLC fueron adquiridos en Tedia High Purity Solvents (Fairfield, OH). Las sales de extracción "Quick, Easy, Cheap, Effective, Rugged, and Safe" (QuEChERS) (Método AOAC) y los kits SPE de dispersión (Bond Elut), fueron adquiridos en Agilent Technologies (Santa Clara, CA, USA).

Preparación y extracción de muestras. De cada muestra se tomaron $7 \mathrm{~g}$ de miel y $3 \mathrm{~g}$ de cera, las cuales previamente fueron descongeladas a temperatura ambiente. Se realizó la extracción de residuos de plaguicidas de acuerdo con una modificación del método analítico "QuEChERS" (Valdovinos-Flores et al., 2017), previamente validado en el Laboratorio de Servicios Analíticos de la Sede Noreste del CIATEJ. Este método consta de dos pasos: (1) la separación de plaguicidas de la matriz con acetonitrilo, y (2) el extracto de limpieza. Se utilizaron curvas de calibración en matriz (matrix-matched calibration), los analitos y el estándar interno se agregaron después de pesar las muestras, antes de agregar disolventes. Se transfirieron $300 \mu \mathrm{L}$ de extracto a viales de 2 $\mathrm{mL}$. Una muestra fue inyectada en un sistema de Cromatografía de líquidos, acoplado a un espectrómetro de masas en tiempo de vuelo (LC-QTOF); y otra en un Cromatógrafo de gases acoplado a un espectrómetro de masas triple cuadrupolo (GC-MS/MS). 
Extracción en miel. De acuerdo al método analítico "QuEChERS" modificado por Valdovinos-Flores et al. (2017), en un tubo de plástico para centrífuga de $50 \mathrm{~mL}$, se pesaron $7 \mathrm{~g}$ de miel, a los cuales se agregaron $10 \mathrm{~mL}$ de agua desionizada; se agitaron manualmente las muestras durante un minuto, se agregaron $15 \mathrm{~mL}$ de acetonitrilo acidificado al $1 \%$ con ácido acético ( $/ \mathrm{v})$ y se agitó nuevamente durante 1 min. Posteriormente se usaron $6 \mathrm{~g}$ de $\mathrm{MgSO}_{4}$ y $1.5 \mathrm{~g}$ de acetato de sodio. Todas las muestras fueron agitadas durante $1 \mathrm{~min}$. y se centrifugaron a 4000 rpm por $5 \mathrm{~min}$.

Para hacer la limpieza del extracto se utilizaron $8 \mathrm{~mL}$ del sobrenadante, y se transfirieron a un tubo de $15 \mathrm{~mL}$, con $400 \mathrm{~mL}$ de amina primaria-secundaria (PSA), $1200 \mathrm{mg}$ de $\mathrm{MgSO}_{4}$ y $400 \mathrm{mg}$ de EC-C18; se agitaron durante $1 \mathrm{~min}$. y se centrifugaron a $4000 \mathrm{rpm}$ por $5 \mathrm{~min}$.

Extracción en cera. Para la extracción de esta matriz fue utilizado el método de Niell et al. (2014). En un tubo de plástico para centrífuga de $50 \mathrm{~mL}$ se pesaron $3 \mathrm{~g}$ de cera, se agregaron $15 \mathrm{~mL}$ de acetonitrilo acidificado al 1\% con ácido acético (v/v). Los tubos se colocaron en un baño de agua a $80{ }^{\circ} \mathrm{C}$ hasta la fundición de la cera. Una vez fundida la cera, se agitaron durante $20 \mathrm{seg}$. y se colocaron nuevamente en el baño para que se funda; se repitió el proceso de fundición y agitación tres veces más. Las muestras se colocaron a temperatura ambiente y luego se llevaron a un congelador a $-20{ }^{\circ} \mathrm{C}$ durante dos horas.

Para hacer la limpieza del extracto, se extrajeron $8 \mathrm{~mL}$ del sobrenadante y se transfirieron a un tubo de $15 \mathrm{~mL}$, con $400 \mathrm{~mL}$ de amina primaria-secundaria (PSA), $1200 \mathrm{mg}$ de $\mathrm{MgSO}_{4}$ y $400 \mathrm{mg}$ de EC-C18. Se agitaron durante $1 \mathrm{~min}$. y se centrifugaron a 4000 rpm por 5 $\min$.

Cromatografía de líquidos acoplado a un espectrómetro de masas en tiempo de vuelo (LC-QTOF). Para el análisis de LC, se utilizó un sistema HPLC de la serie 1200 de Agilent (Agilent Technologies), con una bomba binaria acoplada a un espectrómetro de masas G6530A Q-TOF (Agilent Technologies). La separación cromatográfica se logró utilizando una columna Eclipse Plus C18 (100 mm x $2.1 \mathrm{~mm} \times 1.8 \mu \mathrm{m}$, Agilent Technologies). Las fases móviles consistían en agua con $0.01 \%$ de ácido fórmico +10 $\mathrm{mM}$ de formato de amonio (Disolvente A) y metanol con $0.01 \%$ de ácido fórmico $+10 \mathrm{mM}$ formato de amonio (Disolvente B).

La inyección se realizó utilizando una disolución de automuestrador, en la que se mezclaron $3 \mu \mathrm{L}$ de extracto con $15 \mu \mathrm{L}$ de disolvente $\mathrm{A}$. El gradiente de elución fue el siguiente: $20-50 \%$ B a 0-3.5 min, $50-90 \%$ B a 3.25-8.81 min, 90-100\% B a 8.81-10 min, $100 \%$ B a 10-12.8 min y re-equilibración a condiciones iniciales de 12.9 min a 18 min. Para el análisis de espectrometría de masas, se utilizó una fuente de ionización por electropulverización Agilent Jet-Stream, que operaba en modo iónico positivo, con los siguientes parámetros de operación: modo de adquisición TOF MS, rango de adquisición de $50-950 \mathrm{~m} / \mathrm{z}, \mathrm{N}_{2}$ a $180{ }^{\circ} \mathrm{C}$ y $13 \mathrm{~L} / \mathrm{min}$ como gas de secado, presión del nebulizador a 
40 psi, tensión de la boquilla a $0 \mathrm{~V}$, gas de vaina a $300{ }^{\circ} \mathrm{C}$ y $10 \mathrm{~L} / \mathrm{min}$, tensión capilar a $4000 \mathrm{~V}$, voltaje skimmer a $65 \mathrm{~V}$, voltaje fragmentador a $150 \mathrm{~V}$, octapole RF at $750 \mathrm{~V}$. Agilent Mass Hunter, Workstation se utilizó para la adquisición y análisis de datos.

Cromatografía de gases acoplado a un espectrómetro de masas triple cuadrupolo (GC-MS/MS). Para la cromatografía de gases, se usó un cromatógrafo de gases 7890A, acoplado a un espectrómetro de masas cuadrúpedo triple $7000 \mathrm{~B}$, con ionización de impacto de electrones (EI), equipado con un automuestreador 7693A (Agilent Technologies). La separación cromatográfica se realizó utilizando dos columnas capilares DB-5 MS ultra interés $(15 \mathrm{~m} \times 0.250 \mathrm{~mm} \times 0.25 \mu \mathrm{m}$ de espesor de película; Agilent Technologies). Se utilizó una unión final purgada para conectar las dos columnas, y se realizó un lavado después de cada ejecución. Se inyectaron $2 \mu \mathrm{L}$ del extracto en modo sin divisiones (5 min a $21.1 \mathrm{psi}$ ), con un flujo constante de $1.0 \mathrm{~mL} / \mathrm{min}$ (columna 1 ) y 1.2 $\mathrm{mL} / \mathrm{min}$ (columna 2).

El helio de alta pureza se utilizó como gas portador. La configuración del inyector fue de $65{ }^{\circ} \mathrm{C}$ (contener $0.2 \mathrm{~min}$ ) a $310{ }^{\circ} \mathrm{C}$ a $600{ }^{\circ} \mathrm{C} / \mathrm{min}$; la temperatura del horno se programó de $60{ }^{\circ} \mathrm{C}(1 \mathrm{~min})$ a $170{ }^{\circ} \mathrm{C}$ a $40{ }^{\circ} \mathrm{C} / \mathrm{min}$ a $310{ }^{\circ} \mathrm{C}(4 \mathrm{~min})$. El espectrómetro de masas fue operado en el modo de ionización de impacto de electrones (energía de ionización 70 $\mathrm{eV}$ ); mientras que la línea de trasferencia y las temperaturas de la fuente de iones se fijaron a $300^{\circ} \mathrm{C}$.

Para la selección y cuantificación de análisis se utilizó el modo de monitoreo de iones (SIM), seleccionado con un mínimo de tres iones para cada análisis. La velocidad de escaneo para cada segmento se estableció aproximadamente en dos escaneos, con el fin de obtener un mínimo de 10 puntos de datos por pico.

\section{RESULTADOS}

Cuadro 1. Plaguicidas encontrados en muestras de cera y miel en colonias de abejas melíferas bajo colapso (BC), con antecedentes de colapso (CA) y sin antecedentes de colapso (SA), por LCQTOF y GC-MS/MS

\begin{tabular}{ccccccc}
\hline Grupo & $\begin{array}{c}\text { Tipo de } \\
\text { muestra }\end{array}$ & Insecticidas & Fungicidas & Acaricidas & Herbicidas & Total \\
\hline \multirow{2}{*}{ BC } & Cera & 4 & 1 & 0 & 0 & 5 \\
& Miel & 2 & 1 & 0 & 0 & 3 \\
CA & Cera & 13 & 4 & 1 & 1 & 19 \\
& Miel & 5 & 2 & 0 & 0 & 7 \\
SA & Cera & 11 & 4 & 1 & 1 & 17 \\
& Miel & 5 & 0 & 0 & 0 & 5 \\
& & & & & & \\
& BC & 6 & 2 & 0 & 0 & 8 \\
& CA & 18 & 6 & 1 & 1 & 26 \\
& SA & 16 & 4 & 1 & 1 & 22 \\
\hline
\end{tabular}


Plaguicidas detectados en cera y miel de colonias BC, CA y SA. En la cera se encontró una mayor diversidad de plaguicidas (insecticidas, fungicidas, acaricidas y herbicidas), con respecto a las muestras de miel (insecticidas y fungicidas). Por otra parte, el mayor número de plaguicidas por grupos se encontró en las colonias con antecedentes de colapso (20), seguido por las colonias sin antecedentes (19) y finalmente con aquellas bajo colapso (7). Este mismo comportamiento se observó al separar los plaguicidas por categoría, encontrándose en todos los casos, una mayor diversidad en $\mathrm{CA}$, seguido por SA y finalmente por BC (cuadro 1).

Con respecto a la cantidad de plaguicidas, el insecticida acetamiprid fue el único detectado en todas las muestras de cera analizadas y estuvo presente en una mayor cantidad en las colonias CA $\left(0.686 \mathrm{mg} \mathrm{kg}^{-1}\right)$, seguido por BC $\left(0.402 \mathrm{mg} \mathrm{kg}^{-1}\right)$, y finalmente por SA (0.234 $\mathrm{mg} \mathrm{kg}^{-1}$ ) (cuadro 2). Este mismo insecticida se presentó en todas las muestras de miel, pero en mayor cantidad en las colonias $B C\left(0.633 \mathrm{mg} \mathrm{kg}^{-1}\right)$, seguido por las SA (0.404 $\left.\mathrm{mg} \mathrm{kg}^{-1}\right)$, y finalmente las CA $\left(0.266 \mathrm{mg} \mathrm{kg}^{-1}\right.$ ) (cuadro 3).

Por otro lado, el insecticida malatión se encontró en 11 muestras de cera de las colonias $\mathrm{CB}, \mathrm{CA}$ y $\mathrm{SA}$; sin embargo, las cantidades fueron bajas. Cabe agregar que la permetrina cis se encontró únicamente en seis muestras de cera CA $\left(0.087 \mathrm{mg} \mathrm{kg}^{-1}\right)$ y SA $(0.002 \mathrm{mg}$ $\mathrm{kg}^{-1}$ ) (cuadro 2).

Cuadro 2. Promedio de plaguicidas $\left(\mathrm{mg} \mathrm{kg}^{-1}\right)$ en cera de colonias de abejas melíferas bajo (BC), con (CA) y sin (SA) antecedentes de colapso, detectados por LC-QTOF y GC-MS/MS

\begin{tabular}{|c|c|c|c|c|c|c|c|c|c|c|c|c|c|c|c|c|}
\hline \multirow{2}{*}{ Plaguicio } & \multicolumn{2}{|c|}{ BC } & \multicolumn{5}{|c|}{ CA } & \multicolumn{5}{|c|}{ SA } & \multirow{2}{*}{$\begin{array}{l}\text { Positiva } \\
\text { s }\end{array}$} & \multirow{2}{*}{$\begin{array}{l}B C \\
(\bar{x})\end{array}$} & \multirow{2}{*}{$\begin{array}{l}\text { CA } \\
(\bar{x})\end{array}$} & \multirow{2}{*}{$\begin{array}{l}\text { SA } \\
(\bar{x})\end{array}$} \\
\hline & 1 & 2 & 1 & 2 & 3 & 4 & 5 & 1 & 2 & 3 & 4 & 5 & & & & \\
\hline \multirow[t]{2}{*}{ A } & 0.569 & 0.235 & 1.716 & 0.062 & 0.64 & 0.735 & 0.28 & 0.198 & 0.072 & 0.311 & 0.35 & 0.237 & 12 & 0.402 & 0.686 & 0.234 \\
\hline & CL & CL & CL & CL & CL & CL & CL & CL & CL & CL & CL & CL & & & & \\
\hline \multirow[t]{2}{*}{ B } & & & 0.009 & 0.032 & T & 0.008 & & & & & & & 4 & & & \\
\hline & & & CL & CL & CL & $\mathrm{CL}$ & & & & & & & & & & \\
\hline \multirow[t]{2}{*}{ C } & & & & & & & & 0.006 & & & & & 1 & & & \\
\hline & & & & & & & & $\begin{array}{l}\text { CL- } \\
C G\end{array}$ & & & & & & & & \\
\hline \multirow[t]{2}{*}{ D } & & & 0.025 & 0.024 & 0.023 & 0.025 & 0.025 & & 0.024 & 0.021 & 0.023 & 0.023 & 9 & & 0.025 & 0.023 \\
\hline & & & $\begin{array}{l}\text { CL- } \\
C G\end{array}$ & $\begin{array}{l}\text { CL- } \\
\mathrm{CG}\end{array}$ & $\begin{array}{l}\text { CL- } \\
\mathrm{CG}\end{array}$ & $\begin{array}{l}\text { CL- } \\
\mathrm{CG}\end{array}$ & $\begin{array}{l}\text { CL- } \\
\mathrm{CG}\end{array}$ & & $\begin{array}{l}\text { CL- } \\
\mathrm{CG}\end{array}$ & $\begin{array}{l}\text { CL- } \\
\mathrm{CG}\end{array}$ & $\begin{array}{l}\text { CL- } \\
\mathrm{CG}\end{array}$ & $\begin{array}{l}\text { CL- } \\
\mathrm{CG}\end{array}$ & & & & \\
\hline \multirow[t]{2}{*}{ E } & & & & & & & $\mathrm{T}$ & & & & & & 1 & & & \\
\hline & & & & & & & CL & & & & & & & & & \\
\hline \multirow[t]{2}{*}{$\mathbf{F}$} & & & 0.018 & & 0.009 & & 0.008 & 0.006 & & & & 0.008 & 5 & & 0.012 & 0.007 \\
\hline & & & CL & & CL & & CL & CL & & & & CL & & & & \\
\hline \multirow[t]{2}{*}{ G } & 0.004 & & 0.004 & & 0.007 & & 0.006 & 0.01 & 0.022 & 0.025 & & & 7 & & 0.006 & 0.019 \\
\hline & $\begin{array}{l}\text { CL- } \\
\text { CG }\end{array}$ & & $\begin{array}{l}\text { CL- } \\
\text { CG }\end{array}$ & & $\begin{array}{l}\text { CL- } \\
\text { CG }\end{array}$ & & $\begin{array}{l}\text { CL- } \\
C G\end{array}$ & $\begin{array}{l}\text { CL- } \\
\text { CG }\end{array}$ & $\begin{array}{l}\text { CL- } \\
\text { CG }\end{array}$ & $\begin{array}{l}\text { CL- } \\
\text { CG }\end{array}$ & & & & & & \\
\hline H & & & & & & T & & & & T & & & 2 & & & \\
\hline
\end{tabular}




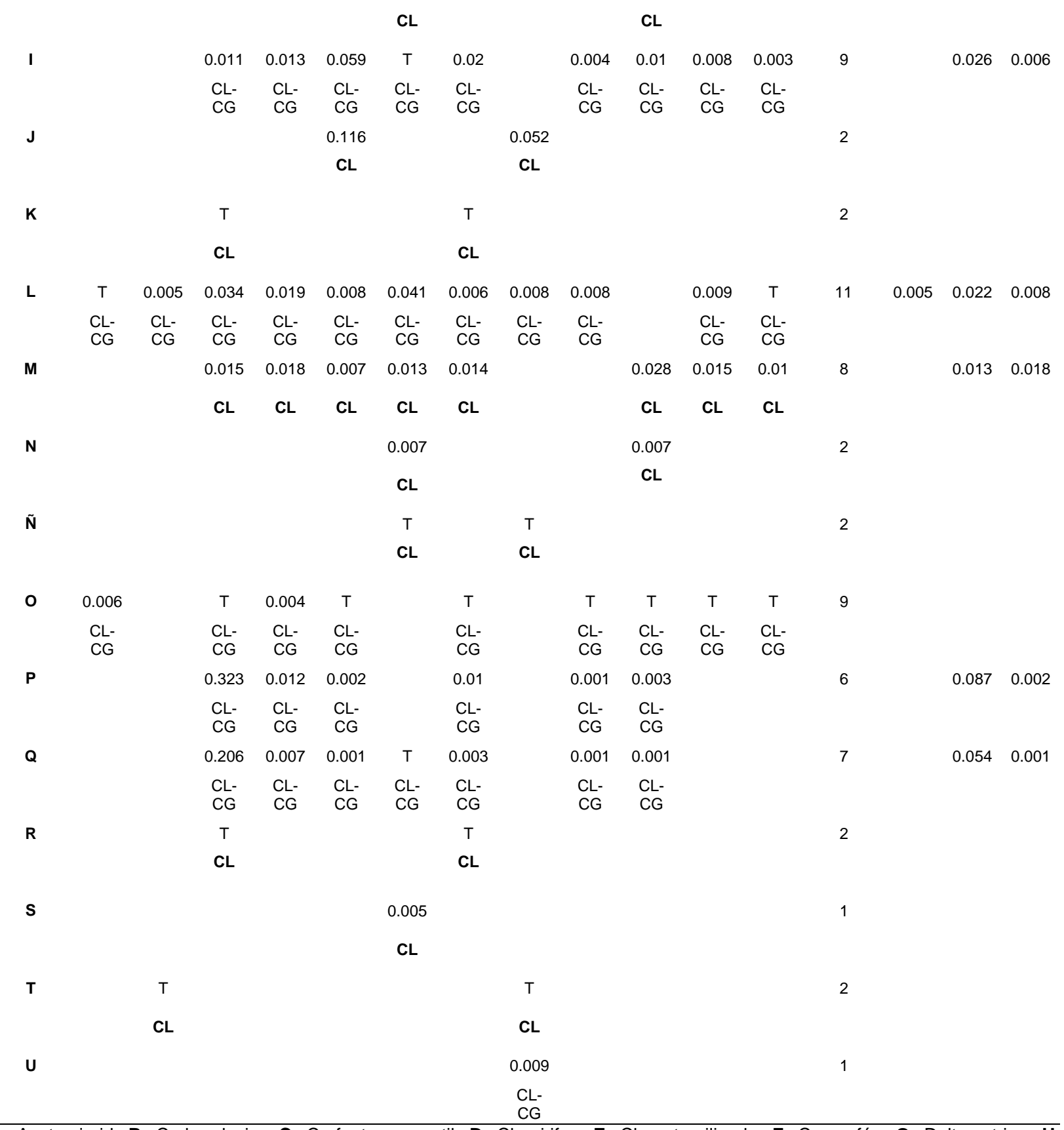

A: Acetamiprid, B: Carbendazim, C: Carfentrazona etil, D: Clorpirifos, E: Clorantraniliprole, F: Coumafós, G: Deltametrina, H: Dimetoato, I: Difenilamina, J: Fluoxastrobina, K: Imidacloprid, L: Malatión, M: Malaoxon, N: Metoxyfenozide, Ñ: Propargita, O: Pentaclorofenol, P: Permetrina, cis-, Q: Permetrina, trans-, R: Pyraclostrobin, S: Tebutiuron, T: Tiofanato, U: Trifloxistrobina T: Trazas, CL: Cromatografía de líquidos CG: Cromatografía de gases

\section{DISCUSIÓN}

Diversidad de plaguicidas en cera y miel. Las muestras de cera presentaron una mayor diversidad de plaguicidas (insecticidas, fungicidas, acaricidas y herbicidas), con respecto a las de miel. De la misma manera Johnson et al. (2010), reportan la presencia ocasional de residuos de plaguicidas en la miel, debido a que la mayoría de los plaguicidas son 
hidrofóbicos y pueden transferirse con mayor facilidad a través de interacciones de las abejas melíferas hacia la cera de éstas (Calatayud-Vernich et al., 2018). Por lo tanto, los plaguicidas se pueden encontrar con mayor frecuencia en la cera que en la miel; o bien, aunque los niveles de plaguicidas en la miel son bajos, éstos tienden a contaminar la cera por su naturaleza lipofílica (Valdovinos-Flores et al., 2017); así como un bajo reemplazo de cera en la colmena y el reciclar para volver a introducir. Por tanto, los plaguicidas de alta hidrofobicidad y estabilidad, son los principales factores para el almacenamiento de plaguicidas en la cera (Calatayud-Vernich et al., 2018).

Cuadro 3. Promedio de plaguicidas $\left(\mathrm{mg} \mathrm{kg}^{-1}\right)$ en miel de colonias de abejas melíferas bajo (BC), con (CA) y sin (SA) antecedentes de colapso, detectados por LC-QTOF

\begin{tabular}{|c|c|c|c|c|c|c|c|c|c|}
\hline \multirow{2}{*}{\multicolumn{2}{|c|}{ Muestra }} & \multicolumn{8}{|c|}{ Plaguicidas en miel } \\
\hline & & A & B & C & D & E & $\mathbf{F}$ & $\mathbf{G}$ & $\mathbf{H}$ \\
\hline \multirow{4}{*}{ BC } & 1 & 0.579 & 0.004 & & & & 0.006 & & \\
\hline & 2 & 0.687 & trazas & & & & & & \\
\hline & 1 & 0.255 & & & & trazas & 0.006 & & \\
\hline & 2 & 0.141 & 0.008 & & trazas & trazas & & & \\
\hline \multirow[t]{5}{*}{ CA } & 3 & 0.105 & & & & & & 0.002 & \\
\hline & 4 & 0.600 & & & & & & 0.004 & \\
\hline & 5 & 0.231 & & 0.008 & & & & 0.003 & \\
\hline & 1 & 0.787 & & & & trazas & 0.007 & 0.005 & \\
\hline & 2 & 0.101 & & & & & & 0.014 & trazas \\
\hline \multirow[t]{7}{*}{ SA } & 3 & 0.290 & & & & trazas & & & \\
\hline & 4 & 0.487 & & & & & 0.009 & & \\
\hline & 5 & 0.353 & & & & & & & \\
\hline & Positivas & 12 & 3 & 1 & 1 & 4 & 4 & 5 & 1 \\
\hline & Promedio BC & 0.633 & & & & & & & \\
\hline & Promedio CA & 0.266 & & & & & & 0.003 & \\
\hline & Promedio SA & 0.404 & & & & & & 0.010 & \\
\hline
\end{tabular}

A: Acetamiprid, B: Carbendazim, C: Imidacloprid, D: Fluoxastrobina, E: Dimetoato, F: Malaoxon, G: Metamidofos, H: Ometoato.

Del total de los plaguicidas, los insecticidas fueron los principales encontrados en las muestras de cera, con el $59.1 \%$, lo que corresponden principalmente a organofosforados (clorpirifos, malatión, coumafós, dimetoato y malaoxon), piretroides (deltametrina, permetrina cis y trans), neonicotinoides (acetamiprid e imidacloprid), y organoclorados (pentaclorofenol). Asimismo, en la miel se encontró organofosforados (dimetoato malaoxon, metamidofos y ometoato), y neonicotinoides (acetamiprid e imidacloprid); 
representando el $75.0 \%$ de las muestras. La presencia de insecticidas en la miel y cera de abejas representa el mayor riesgo para los insectos polinizadores (Botías y SánchezBayo, 2018; Ostiguy et al., 2019); por tanto, la detección y cuantificación de éstos en las muestras analizadas, reflejan su alta exposición de las abejas, ocasionando daños irreversibles en algunas colonias.

Los fungicidas en la cera (27.3\%) y miel (25.0\%), fueron la segunda clase de plaguicidas encontrados con mayor presencia. Botías y Sánchez-Bayo (2018) señalan que algunos fungicidas pueden aumentar la toxicidad de los insecticidas al reducir la capacidad de desintoxicación de las abejas. También se ha encontrado que los residuos de fungicidas en las colonias están relacionados con la prevalencia de enfermedades en las abejas (Simon-Delso et al., 2014). Además, se sugiere que el efecto de los fungicidas en los polinizadores no es por toxicidad directa, sino por la alteración del microbioma presente en el polen y néctar de las plantas tratadas y/o contaminadas de las que se alimentan las abejas y de su propia flora bacteriana (VanEngelsdorp et al., 2009); lo que tiene importantes consecuencias en la nutrición y estado de salud de las abejas.

Finalmente, los herbicidas (9.1\%) y acaricidas (4.5\%), son los plaguicidas con menor presencia en las muestras de cera analizadas. Los herbicidas no representan toxicidad aguda para los insectos polinizadores (Botías y Sánchez-Bayo, 2018); sin embargo, su uso afecta de manera indirecta a las abejas, porque eliminan gran cantidad de plantas silvestres y reducen la diversidad floral, que es la fuente principal de alimento (Bohnenblust et al., 2016); a esto puede atribuirse la baja presencia de herbicidas en las muestras analizadas.

Para el caso de los acaricidas, los cuales son utilizados para el control de Varroa destructor, pueden actuar de manera aditiva o sinérgica con los residuos de insecticidas en las colonias de abejas (Johnson et al., 2013); sin embargo, en nuestro caso, los acaricidas encontrados en las muestras fue del $4.5 \%$ del total de plaguicidas, por lo que posiblemente éstos ocasionan efectos adversos menores en las colonias de abejas.

Diversidad de plaguicidas en cera y miel de colonias BC, CA y SA. Las colonias Con Antecedentes de Colapso tuvieron mayor diversidad de plaguicidas (20), respecto a las colonias Sin Antecedentes (19), y las colonias Bajo Colapso (7); lo cual se refleja también al desagregar a los plaguicidas en insecticidas, fungicidas, acaricidas y herbicidas; excepto para el caso de estos dos últimos, en los que no hubo presencia en $\mathrm{BC}$, y la diversidad fue similar en las colonias CA y SA.

Alcántar-Rosales et al. (2016), reportan en miel y cera datos similares en relación a la reducida diversidad de plaguicidas para las colonias que colapsaron, encontrando dos plaguicidas (neonicotinoides y organofosforados) en las muestras de miel, y cuatro (organofosforados, benzimidazol, piretroides y derivado de la piridina) en la cera; sin embargo, éstos se encuentran en bajas concentraciones. En relación a esto, la cera 
contaminada con plaguicidas, al estar en contacto con el huevo en desarrollo hasta que emerge la abeja, pueden llegar a provocar efectos sub-letales en las abejas obreras, afectando principalmente el desarrollo larvario y la longevidad de las abejas (Wu et al., 2011); pudiendo provocar efectos indirectos en la colonia, tales como cambios prematuros en el rol de las abejas y en la actividad de pecoreo. Para el presente caso, las colonias que colapsaron presentaron la menor diversidad de plaguicidas, pero en altas cantidades; lo que posiblemente demuestra que la presencia de insecticidas y fungicidas afectaron a las abejas, provocando el colapso.

Es importante resaltar además, que la presencia de plaguicidas se debe al manejo de las colmenas (estacionarias y migratorias), así como a la ubicación del apiario, entre otros factores (Ostiguy et al., 2019). Para nuestro caso, en los tres grupos (CA, SA y BC), la mayoría de las colmenas son movilizadas, y se ha reportado que la movilización de colmenas destinadas para la polinización provoca una mayor exposición a plaguicidas (Traynor et al., 2016). En nuestro caso, los apicultores las movilizan, debido a dos razones: la búsqueda de floración, y cuando son arrendadas para la polinización de cultivos; este manejo puede ocasionar estrés a las abejas, volviéndolas más susceptibles a la intoxicación por plaguicidas (Sánchez-Bayo et al., 2016).

La ubicación de los apiarios también es un factor importante, debido al efecto que tendrá en las colmenas la vegetación circundante. Se ha demostrado que la intensificación de la agricultura provoca la pérdida de hábitats naturales; por tanto, los cultivos intensivos y en general la falta de biodiversidad vegetal limitan la cantidad de alimento, lo que provoca una disminución en abundancia y riqueza de polinizadores; así como un impacto en la salud de las abejas melíferas (Kovács-Hostyánszki et al., 2017).

En nuestro caso, la vegetación circundante al momento del muestreo y los cultivos que mayormente se siembran, son los siguientes: para el grupo BC, la vegetación predominante fue de pinabete (Tamarix spp.); y los cultivos cercanos a los apiarios, fueron maíz (Zea mays), alfalfa (Medicago sativa) y algodón (Gossypium spp.). Para el grupo CA, la vegetación predominante fue pinabete (Tamarix spp.) y mezquite (Prosopis laevigata), con cultivos de maíz (Z. mays), sorgo (Sorghum vulgare), algodón (Gossypium spp.), alfalfa ( $M$. sativa), sandía (Citrullus lannatus), melón (Cucumis melo), chile (Capsicum annuum), y calabaza (Cucurbita pepo). Finalmente, para el grupo SA, la principal vegetación predominante fue mezquite ( $P$. laevigata) y pinabete (Tamarix spp.), con cultivos de alfalfa (M. sativa), maíz (Z. mays), sorgo ( $S$. vulgare) y algodón (Gossypium spp.). Esto da evidencia que las colmenas de la región están ampliamente expuestas a plaguicidas, lo que significa que las zonas agrícolas contribuyen a la alta presencia de plaguicidas en los productos de la colmena (Traynor et al., 2016).

Se encontró la mayor diversidad de residuos de plaguicidas en la miel y cera en las colonias CA, pudiera estar relacionada con la cantidad de cultivos cerca de las colonias donde fueron tomadas las muestras; sin embargo, la cantidad de plaguicidas fue menor 
comparado a las colonias en colapso con presencia de altas cantidades de plaguicidas en miel y cera. Lo anterior, posiblemente se debió a que estas colonias presentaron mayor exposición a los cultivos tratados, o bien, debido a una aplicación reciente de plaguicidas durante la permanencia de las colonias con respecto a CA y SA; ya que presentaron menores niveles de plaguicidas, aun estando en la misma región.

Una exposición crónica de las abejas a los plaguicidas a dosis subletales pueden afectar las funciones neurológicas, como la memoria y el comportamiento; síntomas que pueden presentarse antes del colapso de la colmena (Lu et al., 2014); aunado a esto, la exposición a plagas, enfermedades, una mala nutrición, o la interacción entre plaguicidas y patógenos, contribuyen a la mortalidad de las colonias de abejas (Broadrup et al., 2019), lo que posiblemente ocurrió en las colonias BC.

Cantidad de plaguicidas en muestras de miel y cera. El insecticida encontrado en la totalidad de las muestras de miel y cera fue acetamiprid, mismo que además fue el que en promedio tuvo la mayor cantidad, tanto en miel $\left(0.385 \mathrm{mg} \mathrm{kg}^{-1}\right)$, como en cera (0.316 mg kg-1 ${ }^{1}$ ); rebasando los Límites Máximos de Residuos (LMR) de la Unión Europea (UE) $\left(0.05 \mathrm{mg} \mathrm{kg}^{-1}\right)$. Datos reportados por Gaweł et al. (2019) en miel difieren a los nuestros, cuyas concentraciones son bajas y van de 0.001 a $0.13 \mathrm{mg} \mathrm{kg}^{-1}$. También, Da Silva et al. (2015), reportan un promedio de $0.0025 \mathrm{mg} \mathrm{kg}^{-1}$.

Estudios realizados por El Hassani et al., (2008) indican que el consumo de este plaguicida a dosis subletales de $0.1 \mu \mathrm{g} /$ abeja afecta su comportamiento y aprendizaje olfativo, debido a su efecto inmunosupresor (Di Prisco et al., 2013), provocando una mayor susceptibilidad a la infección del microsporidio Nosema (Broadrup et al., 2019). Además, el debilitamiento inmunológico favorece a la propagación del ácaro Varroa en las colonias de abejas melíferas, el cual es fuente de trasmisión de virus (Di Prisco et al., 2016); como el Virus de las Alas Deformes (DWV), el Virus de la Parálisis Aguda Israelí (IAPV), el Virus de la Parálisis Aguda (ABPV) y el Virus de Kashmir (KBV) (Belsky y Joshi, 2019; Brutscher et al., 2016). La combinación de estas enfermedades con los insecticidas neonicotinoides, contribuyen al colapso de las colmenas (Sánchez-Bayo et al., 2016). Además, el acetamiprid puede presentar efectos sinérgicos cuando es combinado con otros plaguicidas (Wang et al., 2019), lo que posiblemente explica el colapso de las colonias en esta región; aunado a que éstas probablemente tenían mayor tiempo de exposición a los plaguicidas.

El segundo plaguicida en frecuencia fue el malatión, que se encontró en 11 muestras de cera con niveles desde 0.005 hasta 0.041 , y un promedio de $0.015 \mathrm{mg} \mathrm{kg-}^{-1}$; cifra que no rebasa los LMR de la UE de $0.05 \mathrm{mg} \mathrm{kg}^{-1}$. Para el norte de México Valdovinos-Flores et al. (2017), reportan la presencia de malatión en el $100 \%$ de las muestras de cera, con niveles que van de 0.006 a $1.532 \mathrm{mg} \mathrm{kg}^{-1}$, con un promedio de $0.018 \mathrm{mg} \mathrm{kg}^{-1}$. El malatión, utilizado en la agricultura como insecticida y acaricida y para el control de plagas urbanas, presenta baja persistencia y alta toxicidad en insectos (Toxnet, 2019). 
Los resultados de este estudio demuestran una alta incidencia del organofosforado malatión en el norte de México, y aunque presenta baja persistencia, es de alta incidencia en las muestras analizadas. Lo anterior probablemente se explique por la aplicación del organofosforado cerca de las colmenas antes de que fueran recolectadas las muestras, y a que como anteriormente se mencionó, que la mayoría de las colmenas se movilizan en busca de floración y se ubican principalmente cerca de los cultivos agrícolas.

Finalmente, la permetrina cis se encontró en menor frecuencia (seis muestras de cera), pero en mayor cantidad $\left(0.087 \mathrm{mg} \mathrm{kg}^{-1}\right)$; sin embargo, la UE no especifica su LMR. Datos similares son reportados por Johnson et al. (2010), con valores de $0.133 \mathrm{mg} \mathrm{kg}^{-1}$. No obstante, este insecticida es altamente tóxico para las abejas, con una DL50 tópica de $0.024 \mu \mathrm{g} / \mathrm{abeja}$ (Piccolomini et al., 2018). La exposición prolongada de los piretroides pueden afectar la inmunidad celular y humoral; así como la disminución en la inmunidad en las abejas (Qi et al., 2019). La permetrina se utiliza principalmente como insecticida y acaricida para el tratamiento de semillas de uso forestal, y para el control de vectores (Toxnet, 2019); y su amplio uso puede ser la razón de su mayor cantidad y presencia en las muestras analizadas, ya que algunas muestras provenían de la cercanía de zonas agrícolas.

Es importante señalar que La Comarca Lagunera ha sido referente en cuanto a la siembra de algodón, cultivo de forrajes para el ganado bovino, y además la producción hortícola se encuentra en aumento (SIAP, 2019); por lo que es una región donde se han utilizado una gran diversidad de plaguicidas, muchos de los cuales presentan efecto residual (Vargas-González et al., 2016). Por lo tanto las prácticas agrícolas inadecuadas y el uso y manejo ineficiente de los plaguicidas (Esquivel-Valenzuela et al., 2019) han creado un serio problema de salud pública, debido a intoxicaciones por agroquímicos, así como para el medio ambiente; destacándose el daño ocasionado a la apicultura por su efecto en el colapso de las colmenas.

\section{CONCLUSIONES}

La mayor cantidad de plaguicidas se encontraron en la cera de las colonias con antecedentes de colapso; así también presentan la mayor diversidad (insecticidas, fungicidas, acaricidas y herbicidas). La presencia de plaguicidas en la miel y cera de las colonias bajo, con, y sin antecedentes de colapso pueden ser la consecuencia de los tratamientos fitosanitarios utilizados en la agricultura, por lo que su presencia puede verse influida por el origen de la muestra, ya que el radio de acción de las abejas es de hasta diez kilómetros. Sin embargo, nuestros datos no nos permiten afirmar que la presencia de plaguicidas sea la principal o única causa del colapso de las colonias; y por lo tanto, se requiere continuar con este tipo de investigaciones para determinar los factores que afectan la salud de las abejas melíferas, como lo es la presencia de plaguicidas, parásitos y enfermedades en la región. 


\section{AGRADECIMIENTOS}

Al Instituto Nacional de Investigaciones Forestales, Agrícolas y Pecuarias por la oportunidad de realizar el doctorado; a la UAAAN por el financiamiento de los proyectos No. 2834 y 2835; al CIATEJ A.C Sede Noreste y al QFB Víctor Alcántar Rosales por el apoyo en el proceso y análisis de las muestras; a los apicultores por las facilidades para la toma de las muestras y aplicación del cuestionario.

\section{LITERATURA CITADA}

ALCÁNTAR-ROSALES VM, Heras-Ramírez ML, Valdovinos-Flores C, Saldaña-Loza LM, Reyes-Carrillo JL, Dorantes-Ugalde JA, Gaspar-Ramírez O. 2016. Current Situation of Pesticide Use in Mexico and Its Relationship with Colony Collapse Disorder, an Emerging Problem. XVI International Congress of Toxicology. Mérida, México.

BALBUENA MS, Tison L, Hahn ML, Greggers U, Menzel R, Farina WM. 2015. Effects of Sublethal Doses of Glyphosate on Honeybee Navigation. Journal of Experimental Biology. 218: 2799-2805. https://doi.org/ 10.1242/jeb.117291

BELSKY J, Joshi NK. 2019. Impact of biotic and abiotic stressors on managed and feral bees. Insect 10 (233): 1-42. https://doi.org/ 10.3390/insects10080233.

BENUSZAK J, Laurent M, Chauzat MP. 2017. The exposure of honey bees (Apis mellifera; Hymenoptera: Apidae) to pesticides: Room for improvement in research. Science of The Total Environment. 587-588:423-438. https://doi.org/10.1016/J.SCITOTENV.2017.02.062

BOHNENBLUST EW, Vaudo AD, Egan JF, Mortensen DA, Tooker JF. 2016. Effects of the herbicide dicamba on nontarget plants and pollinator visitation. Environmental Toxicology and Chemistry. 35(1):144-51. https://doi.org/10.1002/etc.3169

BOTÍAS C, Sánchez-Bayo F. 2018. Papel de los plaguicidas en la pérdida de polinizadores. Ecosistemas. 27(2):34-41. https://doi.org/10.7818/ECOS.1314

BROADRUP RL, Mayack C, Schick SJ, Eppley EJ, White HK, y Macherone A. 2019. Honey bee (Apis Mellifera) exposomes and dysregulated metabolic pathways associated with Nosema ceranae infection. PLOS ONE 14 (4): 1-19. https://doi.org/10.1371/journal.pone.0215166

BRUTSCHER LM, McMenamin AJ, Flenniken ML. 2016. The Buzz about Honey Bee Viruses. PLoS Pathogens. 12(8):1-7. https://doi.org/10.1371/journal.ppat.1005757

CALATAYUD-VERNICH P, Calatayud, F, Simó E, Picó Y. 2018. Pesticide residues in honey bees, pollen and beeswax: Assessing beehive exposure. Environmental Pollution. 241:106-114. https://doi.org/10.1016/j.envpol.2018.05.062

CRESSWELL JE, Thompson HM. 2012. Comment on A Common Pesticide Survival in Honey Bees. Science. 337:1453-b. https://doi.org/10.1126/science.1224618

DA SILVA PI, Oliveira FAS, Pedroza HP, Gadelha ICN, Melo MM, Soto-Blanco B. 2015. Pesticide exposure of honeybees (Apis Mellifera) pollinating melon crops. Apidologie. 46 
(6): 703-15. https://doi.org/10.1007/s13592-015-0360-3

DI PRISCO G, Annoscia D, Margiotta M, Ferrara R, Varricchio P, Zanni V, Caprio E, Nazzi F, Pennacchio F. 2016. A mutualistic symbiosis between a parasitic mite and a pathogenic virus undermines honey bee immunity and health. Proceedings of the National Academy of Sciences. 113: 1-6. https://doi.org/10.1073/pnas.1523515113

DI PRISCO G, Cavaliere V, Annoscia D, Varricchio P, Caprio E, Nazzi F, Gargiulo G, Pennacchio F. 2013. Neonicotinoid clothianidin adversely affects insect immunity and promotes replication of a viral pathogen in honey bees. Proceedings of the National Academy of Sciences. 110: 18466-18471. https://doi.org/10.1073/pnas.1314923110

EL HASSANI AK, Dacher M, Gary V, Lambin M, Gauthier M, Armengaud C. 2008. Effects of sublethal doses of acetamiprid and thiamethoxam on the behavior of the honeybee (Apis mellifera). Archives of Environmental Contamination and Toxicology. 54(4):653-661. https://doi.org/10.1007/s00244-007-9071-8

ESQUIVEL-VALENZUELA B, Cueto-Wong JA, Valdez-Cepeda RD, Pedroza-Sandoval A, Trejo-Calzada R, Pérez-Veyna O. 2019. Prácticas de manejo y análisis de riesgo por el uso de plaguicidas en La Comarca Lagunera, México. Revista Internacional de Contaminacion Ambiental. 35(1):25-33. https://doi.org/10.20937/RICA.2019.35.01.02

GAWEŁ M, Kiljanek T, Niewiadowska A, Semeniuk S, Goliszek M, Burek O, Posyniak A. 2019. Determination of neonicotinoids and 199 other pesticide residues in honey by liquid and gas chromatography coupled with tandem mass spectrometry. Food Chemistry. 282: 36-47. https://doi.org/10.1016/j.foodchem.2019.01.003

JOHNSON RM, Dahlgren L, Siegfried BD, Ellis MD. 2013. Acaricide, Fungicide and Drug Interactions in Honey Bees (Apis mellifera). PLoS ONE. 8(1):e54092. https://doi.org/10.1371/journal.pone.0054092

JOHNSON RM, Ellis MD, Mullin CA, Frazier M. 2010. Pesticides and honey bee toxicity - USA. Apidologie. 41:312-331. https://doi.org/10.1051/apido/2010018

KOVÁCS-HOSTYÁNSZKI A, Espíındola A, Vanbergen AJ, Settele J, Kremen C y Dicks LV. 2017. Ecological intensification to mitigate impacts of conventional intensive land use on pollinators and pollination. Ecology Letters. 20: 673-89. https://doi.org/10.1111/ele.12762

LOZANO A, Hernando MD, Uclés S, Hakme E, Fernández-Alba AR. 2019. Identification and measurement of veterinary drug residues in beehive products. Food Chemistry. 274: 61-70. https://doi.org/10.1016/j.foodchem.2018.08.055

LU C, Warchol KM, Callahan RA. 2014. Sub-lethal exposure to neonicotinoids impaired honey bees winterization before proceeding to colony collapse disorder. Bulletin of Insectology. 67(1):125-130. https://doi.org/http://www.bulletinofinsectology.org

NIELL S, Hepperle J, Doerk D, Kirsch L, Kolberg D. 2014. QuEChERS-Based Method for the Multiresidue Analysis of Pesticides in Beeswax by LC-MS/MS and GC×GC-TOF. Journal of Agricultural and Food Chemistry. 62:3675-3683. 
https://doi.org/10.1021/jf405771t

O'NEAL ST, Anderson TD, Wu-Smart JY. 2018. Interactions between pesticides and pathogen susceptibility in honey bees. Current Opinion in Insect Science. 26: 57-62. https://doi.org/10.1016/J.COIS.2018.01.006

OLLERTON J. 2017. Pollinator diversity: distribution, ecological function, and conservation. Annual Review of Ecology, Evolution and Sistematics. 48: 353-76. https://doi.org/10.1146/annurev-ecolsys-110316-022919

OSTIGUY N, Drummond FA, Aronstein K, Eitzer B, Ellis JD, Spivak M, Sheppard WS. 2019. Honey bee exposure to pesticides: A four-year nationwide study. Insects. 10(1):134. https://doi.org/10.3390/insects 10010013

PICCOLOMINI AM, Whiten RS, Flenniken ML, O'Neill KM, Peterson RKD. 2018. Acute toxicity of permethrin, deltamethrin, and etofenprox to the alfalfa leafcutting bee. Journal of Economic Entomology. 111 (3): 1001-5. https://doi.org/10.1093/jee/toy014

QI S, Niu X, Wang DH, Wang C, Zhu L, Xue X, Zhang Z, Wu L. 2019. Flumethrin at sublethal concentrations induces stresses in adult honey bees (Apis mellifera L.). Science of the Total Environment. 700. https://doi.org/10.1016/j.scitotenv.2019.134500

SÁNCHEZ-BAYO F, Goulson D, Pennacchio F, Nazzi F, Goka K, Desneux N. 2016. Are bee diseases linked to pesticides? A brief review. Environment International. 89-90:7-11. https://doi.org/10.1016/j.envint.2016.01.009

SIAP. Servicio de Información Agropecuaria y Pesquera. 2018. Abejas población apícola. Disponiblle en: https://www.gob.mx/siap/acciones-y-programas/produccion-pecuaria.

SIAP. Servicio de Información Agroalimentaria y Pesquera) 2019. Coahuila Infografía agroalimentaria 2018. file:///E:/Plaguicidas/Coahuila-Infografia-Agroalimentaria-2018.pdf.

SIMON-DELSO N, Martin GS, Bruneau E, Minsart LA, Mouret C, Hautier L. 2014. Honeybee colony disorder in crop areas: The role of pesticides and viruses. PLOS ONE. 9(7):1-16. https://doi.org/10.1371/journal.pone.0103073

TOSI S, Démares FJ, Nicolson SW, Medrzycki P, Pirk CWW, Human H. 2016. Effects of a neonicotinoid pesticide on thermoregulation of African honey bees (Apis mellifera scutellata). Journal of Insect Physiology. 93:56-63. https://doi.org/10.1016/j.jinsphys.2016.08.010

$\begin{array}{llll}\text { TOXNET (Toxicology } & \text { Data } & \text { Network). }\end{array}$ https://toxnet.nlm.nih.gov/newtoxnet/hsdb.htm

TRAYNOR KS, Pettis JS, Tarpy DR, Mullin CA, Frazier JL, Frazier M, vanEngelsdorp D. 2016. In-hive Pesticide Exposome: Assessing risks to migratory honey bees from in-hive pesticide contamination in the Eastern United States. Scientific Reports. 6(1). https://doi.org/10.1038/srep33207

VALDOVINOS-FLORES C, Gaspar-Ramırez O, Dorantes-Ugalde JA. 2017. Agricultural pesticide residues in honey and wax combs from Southeastern, Central and Northeastern Mexico. Journal of Apicultural Research. 56(5):667-679. https://doi.org/ 
10.1080/00218839.2017.1340798

VANENGELSDORP D, Evans JD, Donovall L, Mullin C, Frazier M, Frazier J, Tarpy DR, Hayes J, Pettis JS. 2009. Entombed Pollen: A new condition in honey bee colonies associated with increased risk of colony mortality. Journal of Invertebrate Pathology 101 (2):147-149. https://doi.org/10.1016/j.jip.2009.03.008

VARGAS-GONZÁLEZ G, Alvarez-Reyna P, Guigón-López V, Cano-Ríos P, Jiménez-Díaz F, Vásquez-Arroyo J, García-Carrillo M. 2016. Patrón de uso de plaguicidas de alto riesgo en el cultivo de melón (Cucumis melo L.) en La Comarca Lagunera. Ecosistemas y Recursos Agropecuarios. 3(9):135 https://doi.org/10.1007/978-3-540-71095-0_2550

WANG Y, Cheng ZY, Li W. 2019. Interaction patterns and combined toxic effects of acetamiprid in combination with seven pesticides on honey bee (Apis Mellifera L.). Ecotoxicology and Environmental $\quad$ Safety. 190. https://doi.org/10.1016/J.ECOENV.2019.110100

WILMART O, Legre A, Graaf DC, Steurbaut W, Delahaut P, Gustin P, Nguyen BK, Saegerman C. 2016. Residues in Beeswax: A Health Risk for the Consumer of Honey and Beeswas?. Journal of Agricultural and Food Chemistry. https://doi.org/10.1021/acs.jafc.6b02813

WU JY, Anelli CM, Sheppard WS. 2011. Sub-lethal effects of pesticide residues in brood comb on worker honey bee (Apis mellifera) development and longevity. PLoS ONE. 6(2). https://doi.org/10.1371/journal.pone.0014720

Publica tus resultados de investigaciones en las revistas abanico. 